\title{
Espessura macular e acuidade visual na retinopatia diabética tratada por panfotocoagulação
}

\author{
Visual acuity and macular thickness in diabetic retinopathy treated \\ with panphotocoagulation
}

\author{
Otacílio de Oliveira Maia Júnior ${ }^{1}$ \\ Walter Yukihiko Takahashi ${ }^{2}$ \\ Yoshitaka Nakashima ${ }^{3}$
}

Trabalho desenvolvido no Serviço de Retina do Hospital das Clínicas da Faculdade de Medicina da Universidade de São Paulo - USP - São Paulo (SP) - Brasil.

Doutor pela Faculdade de Medicina da Universidade de São Paulo - USP - São Paulo (SP) - Brasil.

Chefe do Setor de Retina do Departamento de Oftalmologia do Hospital das Clínicas da Faculdade de Medicina da USP - São Paulo (SP) - Brasil.

Médico Assistente e Doutor do Departamento de Oftalmologia do Hospital das Clínicas da Faculdade de Medicina da USP - São Paulo (SP) - Brasil.

Endereço para correspondência: Otacílio de Oliveira Maia Júnior. Rua Adelmário Pinheiro, 10 - Salvador (BA) CEP 41900-540

E-mail: omaiausp@uol.com.br

Recebido para publicação em 04.02 .2007

Última versão recebida em 19.06.2007

Aprovação em 25.06.2007

Nota Editorial: Depois de concluída a análise do artigo sob sigilo editorial e com a anuência do Dr. Roberto Lauande Pimentel sobre a divulgação de seu nome como revisor, agradecemos sua participação neste processo.

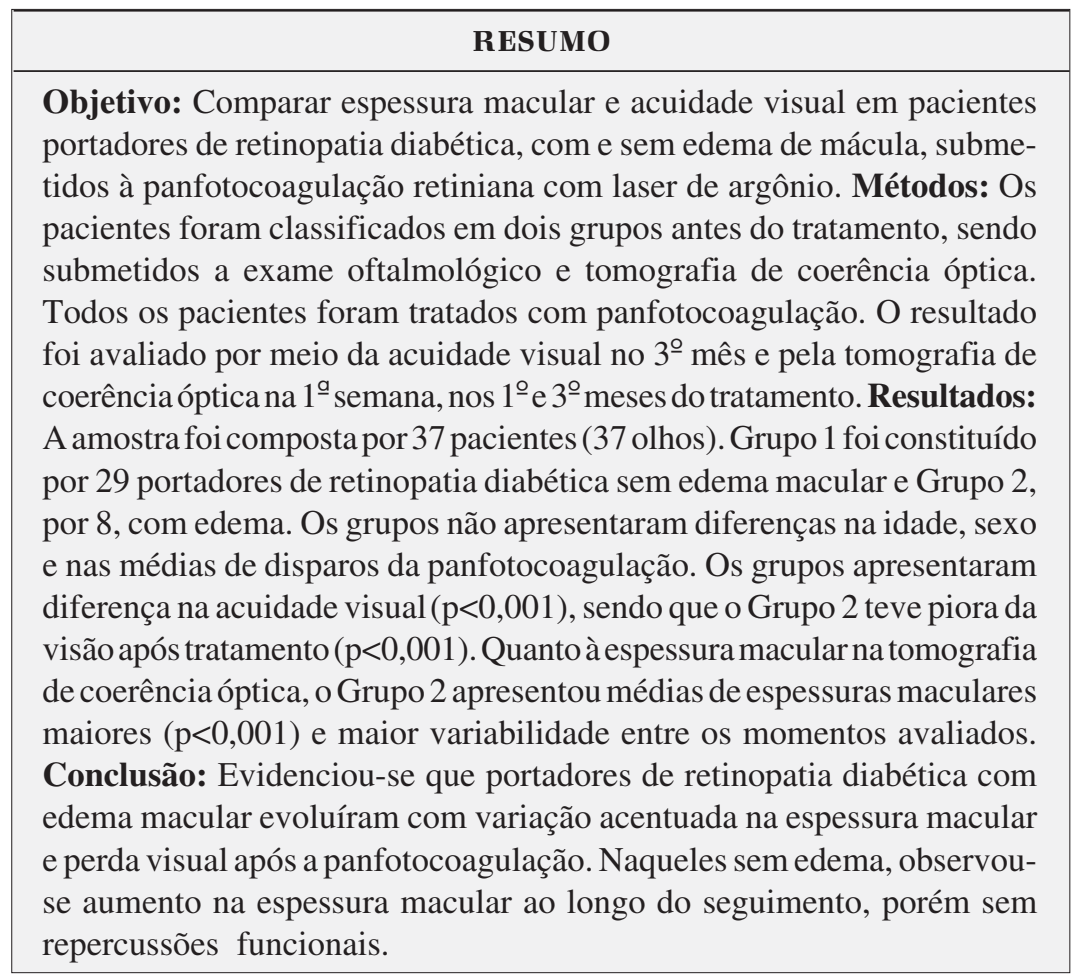

Descritores: Retinopatia diabética; Retinopatia diabética/terapia; Edema macular cistóide; Coagulação por laser; Diabetes mellitus/complicações; Acuidade visual; Tomografia de coerência óptica

\section{INTRODUÇÃO}

O "Early Treatment Diabetic Retinopathy Study" (ETDRS) definiu as estratégias do tratamento da retinopatia diabética (RD) por meio da fotocoagulação a laser de acordo com sua classificação ${ }^{(1)}$, bem como a técnica de aplicação recomendada ${ }^{(2-3)}$. Segundo este estudo, a panfotocoagulação da retina é indicada para RD não proliferativa grave ou muito grave e para RD proliferativa ${ }^{(3)}$.

A panfotocoagulação retiniana tem sido efetiva na redução da perda visual e prevenção do aparecimento subseqüente de neovascularização em olhos com RD grave ${ }^{(4-6)}$.

Contudo, há riscos de induzir baixa da acuidade visual (AV) e edema macular, que podem piorar após o tratamento, acarretando baixa transitória ou permanente da visão ${ }^{(7-10)}$. O "Diabetes Retinopathy Study" (DRS) evidenciou 
que olhos com edema macular, submetidos à panfotocoagulação para RD não proliferativa ou proliferativa, têm duas vezes mais chances de perda visual do que aqueles sem edema ${ }^{(4,11-12)}$.

O presente estudo objetiva comparar a AV e a espessura macular em portadores de RD com e sem edema macular clinicamente significativo (EMCS) submetidos à panfotocoagulação com laser de argônio.

\section{MÉTODOS}

Foram incluídos pacientes do Departamento de Oftalmologia do Hospital das Clínicas da Faculdade de Medicina da Universidade de São Paulo (HC-FMUSP) portadores de RD com indicação primária de panfotocoagulação retiniana, conforme critérios do ETDRS, e avaliados prospectivamente no período de março a dezembro de 2005. Outros critérios de inclusão: indivíduos de ambos os sexos, qualquer idade, sem tratamento retiniano prévio e sem outras doenças maculares. Os portadores de catarata, opacidades na córnea e complicações da RD (hemorragia vítrea e/ou descolamento de retina), prévias ou durante seguimento, foram excluídos.

Os pacientes foram divididos em dois grupos antes do tratamento: Grupo 1 - pacientes não portadores de EMCS e Grupo 2 - pacientes portadores de EMCS. Considerou-se apenas um olho de cada paciente, sendo submetido a exame oftalmológico completo, incluindo retinografia e angiografia fluoresceínica. Os exames utilizados para avaliar os objetivos do estudo foram: a medida da AV (tabela de Snellen) e a medida da espessura macular por meio da tomografia de coerência óptica (OCT).

Todos os pacientes foram submetidos à panfotocoagulação retiniana com laser de argônio (Ophthalas 532 Eyelite, Alcon Surgical), segundo padronização recomendada pelo ETDRS. O Grupo 2, de portadores de EMCS, foi submetido a laser focal durante o $1^{\circ}$ episódio da panfotocoagulação. Finalizados os 3 episódios de tratamento, os pacientes foram reavaliados por meio de exame de AV com a melhor correção (após 3 meses da panfotocoagulação) e de OCT (na primeira semana, no primeiro e terceiro meses do tratamento). Foi selecionado um caso de cada grupo para ilustração do "follow-up" (Figuras 1 e 2).

As imagens obtidas pelo OCT Stratus version 4.0 software (Carl Zeiss Meditec) foram realizadas no programa de medida da espessura da retina (retinal thickness) e o cursor foi colocado de acordo com a fixação do paciente ou manualmente, no centro da fóvea, quando a depressão foveal era visível. A estratégia para análise utilizada foi o mapa de espessura macular (macular thickness map), mensurada na região central da retina.

No intuito de caracterizar o controle sistêmico, foram solicitados hemoglobina glicosilada $\left(\mathrm{Hb}_{\mathrm{Alc}}\right)$ no pré e pós-tratamento (Método Certificado pelo NGSP - "National Glycohemoglobin Standardization Program", Valor de Referência=4,1 a 6,5\%).

Inicialmente todas as variáveis foram analisadas descritivamente. Para as variáveis quantitativas, esta análise foi feita através da observação dos valores mínimos e máximos e do cálculo de médias, desvios-padrão e medianas. Para as variáveis qualitativas, calcularam-se freqüências absolutas e relativas. Para a análise da hipótese de igualdade entre os grupos, utilizou-se o teste $\mathrm{t}$ de Student ${ }^{(13)}$. Para testar a homogeneidade dos grupos em relação às proporções, foi utilizado o teste exato de Fisher ${ }^{(13)}$. Para averiguar o comportamento dos grupos, considerando as condições estudadas, fez-se uso da técnica Análise de Variância com medidas repetidas ${ }^{(14)}$. O nível de significância utilizado para os testes foi de $5 \%$.

O estudo foi aprovado pela Comissão de Ética para Análise de Projetos e Pesquisa da Diretoria Clínica do HC-FMUSP e todos os pacientes assinaram o termo de consentimento livre e esclarecido.

\section{RESULTADOS}

As características demográficas e clínicas estão demonstradas na tabela 1. A amostra foi composta por 37 pacientes (37 olhos), sendo todos portadores de diabetes mellitus tipo 2 . $\mathrm{O}$ Grupo 1 foi constituído por 29 pacientes e o Grupo 2, por 8 pacientes. Quanto ao olho em estudo, o Grupo 1 teve 13 (44,8\%) e o Grupo 2, $5(62,5 \%)$ olhos direitos tratados. Os dois grupos não apresentaram diferença estatisticamente significante na idade ( $p=0,62)$, no sexo e no olho em estudo.

Quanto ao tipo de retinopatia, houve diferença significativa em relação à classificação. No grupo 2, 100\% apresentavam RD proliferativa e no grupo $1,34,5 \%$ com RD proliferativa e $65,5 \%$ com RD não proliferativa muito grave. Os grupos não apresentaram diferenças estatisticamente significantes no comportamento da $\mathrm{Hb}_{\mathrm{Alc}}$ ao longo das avaliações $(\mathrm{p}=0,59)$, nas médias comparativas entre os mesmos $(p=0,27)$ e nos períodos pré e pós-tratamento $(\mathrm{p}=0,21)$. Quanto à panfotocoagulação retiniana, os grupos não apresentaram diferenças significantes nas médias de disparos do laser no $1^{\circ}, 2^{\circ}$ e $3^{\circ}$ episódios $(\mathrm{p}=0,60)$.

A descrição quantitativa da AV e espessura macular no OCT está demonstrada na tabela 2. Os grupos apresentaram diferenças significantes no comportamento da AV ao longo das avaliações $(\mathrm{p}<0,001)$, sendo que o grupo 1 não apresentou alteração na acuidade após a panfotocoagulação $(\mathrm{p}=0,08)$ e o grupo 2 teve piora da visão $(\mathrm{p}<0,001)$ (Gráfico 1$)$. Os grupos não apresentaram diferenças no comportamento da espessura macular $(\mathrm{p}=0,26)$, sendo observado aumento nas medidas de espessuras ao longo das avaliações nos dois grupos; entretanto, o Grupo 2 apresentou espessuras significantemente maiores nas médias pré, 1 semana, 1 mês e 3 meses do tratamento $(\mathrm{p}<0,001)$ com maior variabilidade em relação ao Grupo 1. Houve alteração nas medidas de espessuras entre os momentos avaliados nos dois grupos $(\mathrm{p}<0,001)$, sendo que o período pré-tratamento difere dos demais momentos: $1^{\underline{a}}$ semana $(p<0,001), 1^{\circ}$ mês $(p=0,017)$ e $3^{\circ}$ mês $(\mathrm{p}=0,005)($ Gráfico 2). 

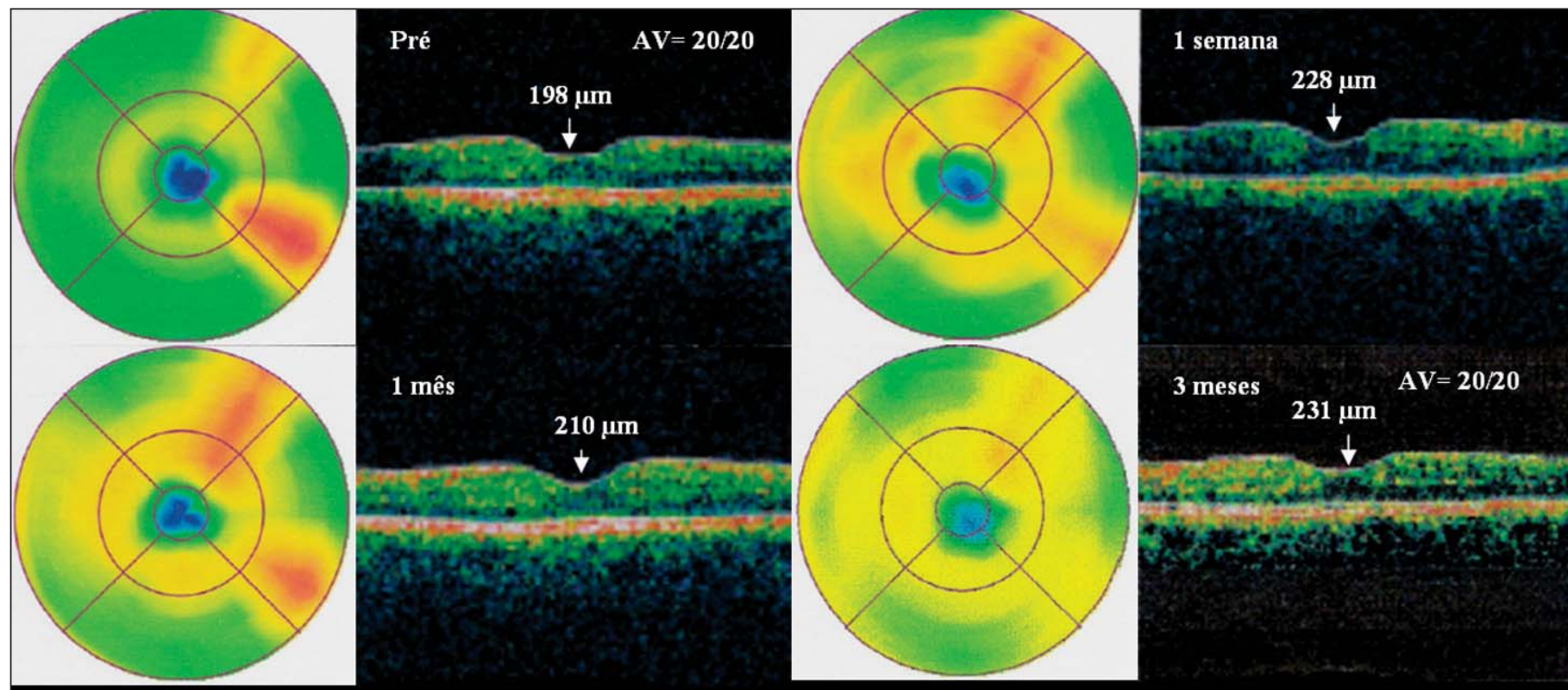

1 mês
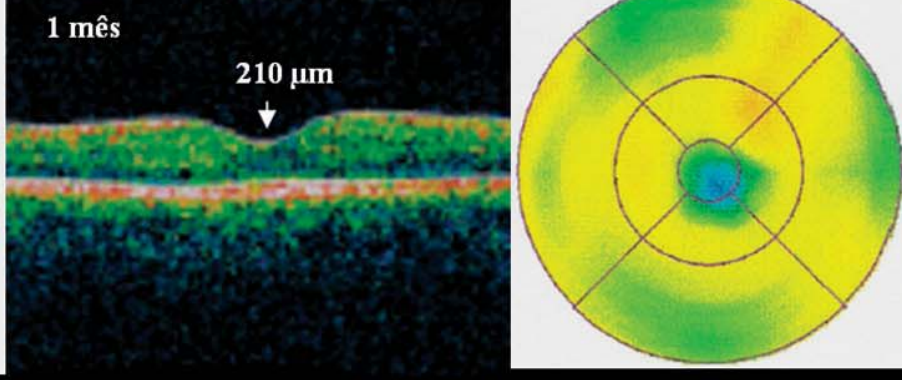

3 meses

$231 \mu \mathrm{m}$

$\mathrm{AV}=\mathbf{2 0 / 2 0}$
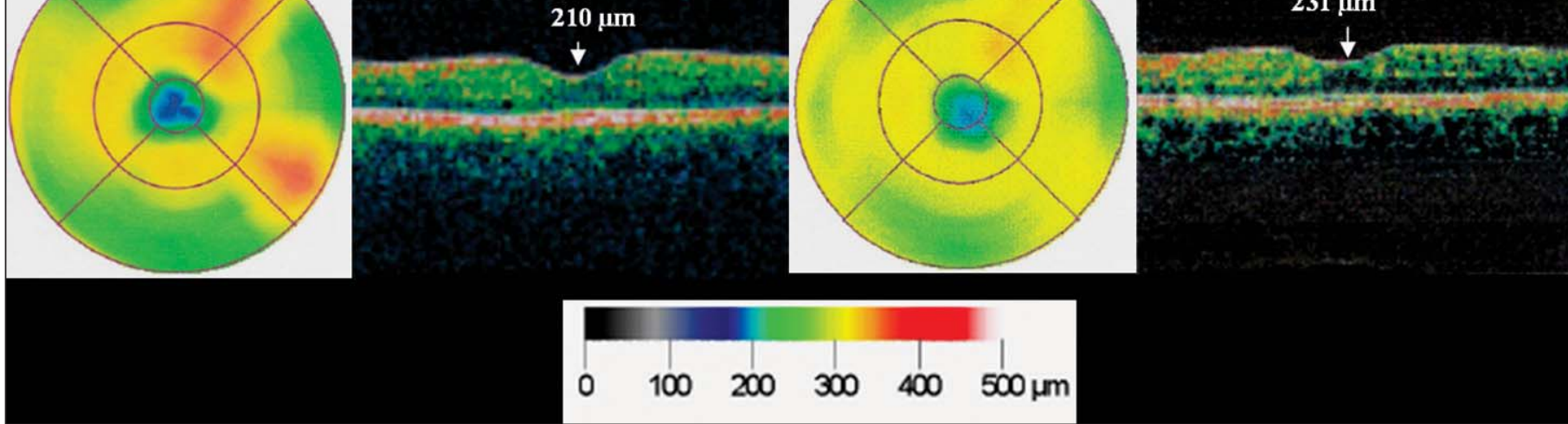

Figura 1 - Paciente do Grupo 1, sexo feminino, 47 anos, submetida à panfotocoagulação no OE por retinopatia diabética não proliferativa muito grave. Observa-se variação subclínica da espessura macular. Acuidade visual não altera no "follow-up".

\section{DISCUSSÃO}

McDonald e Schartz evidenciaram por meio de angiofluoresceinografia que 25 a $43 \%$ dos olhos com RD proliferativa tratados com panfotocoagulação retiniana desenvolvem edema macular ${ }^{(7)}$. Outros autores demonstraram edema subclínico após panfotocoagulação em pacientes sem EMCS prévio ao tratamento por meio do "retinal thickness analyzer" ${ }^{(15)}$ e do $\mathrm{OCT}^{(16)}$. Mais recentemente, foi evidenciado que nos portadores de RD não proliferativa ou proliferativa precoce sem EMCS e submetidos à panfotocoagulação, a perda visual pós-tratamento tem relação com a espessura macular prévia, ou seja, aqueles com espessura parafoveal maior que 300 micra tiveram acuidade visual reduzida após seis meses da fotocoagulação retiniana, denotando que a medida da espessura pelo OCT pode ser um fator preditivo da acuidade visual pós-tratamento ${ }^{(17)}$. No entanto, na literatura pesquisada, não há estudos comparativos avaliando de forma quantitativa o efeito da laserterapia em portadores de RD submetidos à panfotocoagulação com EMCS prévio ao tratamento.

No presente estudo, apesar dos grupos apresentarem aumento na espessura macular, o grupo com EMCS teve maior variabilidade que no grupo sem edema. A mensuração objetiva da espessura da retina, bem como estudo morfológico da interface vítreo-retiniana e das alterações neurossensoriais na maculopatia diabética por meio do OCT vêm se tornando método propedêutico importante para documentação, decisão terapêutica e avaliação do tratamento estabelecido. Alguns autores estabeleceram padrões de espessamento no OCT para o EMCS ${ }^{(18)}$. Recentemente, outros autores demonstraram alta sensibilidade e reprodutibilidade do OCT em detectar EMCS $^{(19)}$. Outros autores mostraram boa correlação entre a biomicroscopia de fundo e OCT para diagnóstico de EMCS em espessuras maiores que 325 micra $^{(20)}$. Entretanto, são poucos trabalhos que abordam quantitativamente o aumento da espessura macular de pacientes com RD submetidos à panfotocoagulação. Nesse estudo, utilizou-se o OCT para avaliar comportamento da espessura macular após a panfotocoagulação em portadores de RD com e sem edema macular, havendo aumento significativo no seguimento de ambos os grupos, mas maior variabilidade no grupo com edema. Isto denota um maior acometimento anatômico naqueles com espessura macular previamente aumentada. Vale ressaltar que o tratamento com laser focal para o EMCS no Grupo 2 foi realizado no primeiro episódio da panfotocoagulação por questões operacionais, não sendo feito antes de iniciar a panfotocoagulação como recomenda o ETDRS, o que pode ter influenciado na variabilidade da espessura macular nesse grupo. No entanto, muitas vezes, não se pode retardar a panfotocoagulação em casos avançados de RD proliferativa, os quais podem evoluir com as complicações da RD e conseqüências limitantes na função visual.

Outra evidência demonstrada foi que no grupo portador de RD com EMCS prévio à panfotocoagulação retiniana houve 


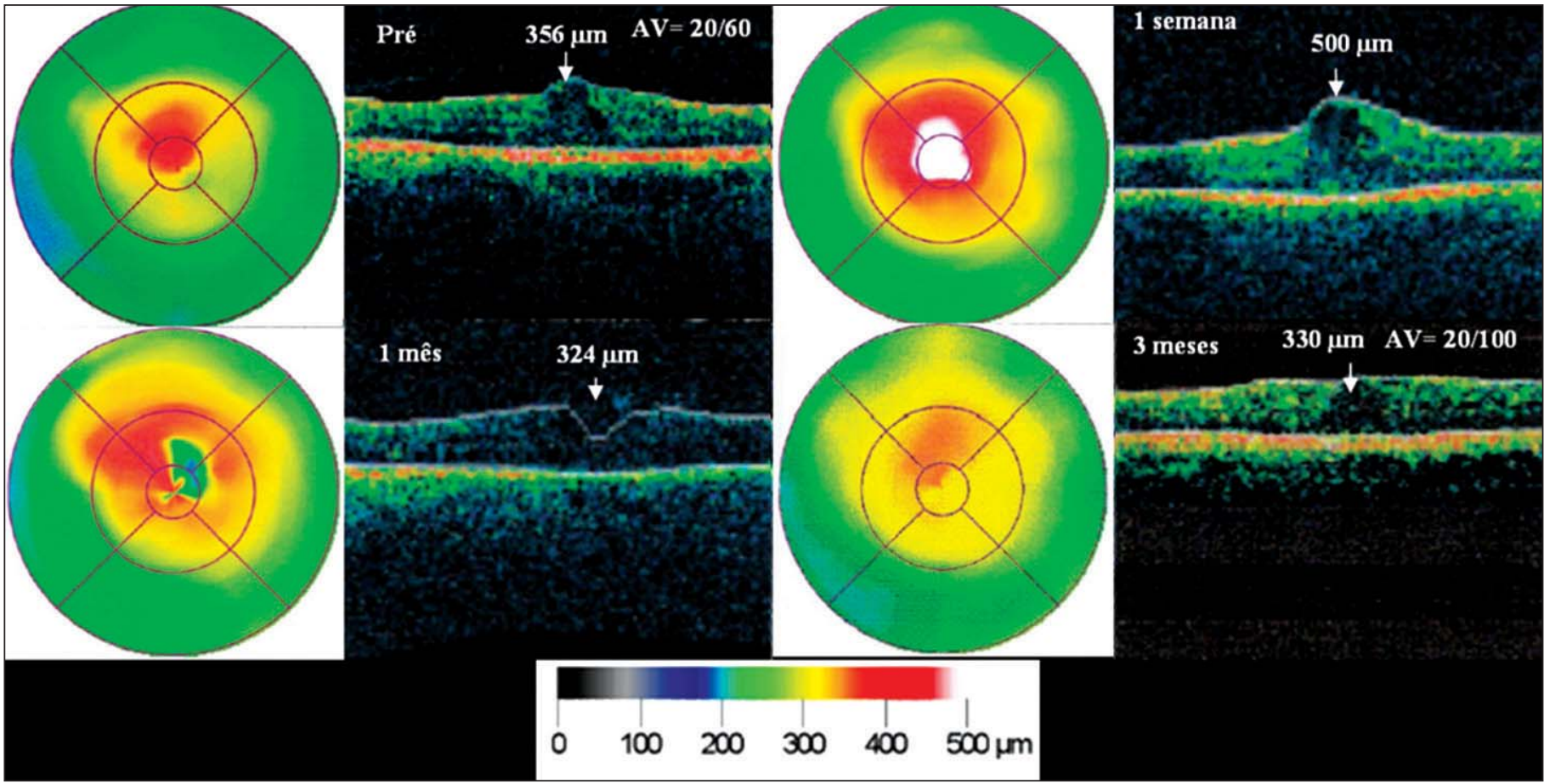

Figura 2 - Paciente do Grupo 2, sexo feminino, 64 anos, submetida à panfotocoagulação no OD por retinopatia diabética proliferativa. Observase variação significante na espessura macular e piora na acuidade visual após 3 meses do tratamento.

Tabela 1. Características de portadores de retinopatia diabética sem edema macular (Grupo 1) e com edema (Grupo 2), submetidos à panfotocoagulação com laser de argônio. Hospital das Clínicas da Faculdade de Medicina da Universidade de São Paulo - 2005

\begin{tabular}{|c|c|c|c|c|c|c|c|c|c|}
\hline \multirow[t]{2}{*}{ Grupo } & \multirow[t]{2}{*}{$n$} & \multirow[t]{2}{*}{ Idade (anos) } & \multicolumn{2}{|c|}{ Sexo } & \multirow[t]{2}{*}{$\mathrm{Hb}_{\mathrm{A} 1 \mathrm{c}}$ - pré } & \multirow[t]{2}{*}{$\mathrm{Hb}_{\mathrm{A} 1 \mathrm{c}}$ - pós } & \multicolumn{2}{|c|}{ Classificação RD } & \multirow{2}{*}{$\begin{array}{c}\text { Disparos } \\
\text { laser }\end{array}$} \\
\hline & & & $f$ & $\%$ & & & $f$ & $\%$ & \\
\hline 1 & 29 & $55,3 \pm 6,3$ & $\begin{array}{l}12 \mathrm{M} \\
17 \mathrm{~F}\end{array}$ & $\begin{array}{l}41,4 \\
58,6\end{array}$ & $8,8 \pm 1,47 \%$ & $8,6 \pm 1,20 \%$ & $\begin{array}{l}10 \text { RDP } \\
19 \text { RDNP }\end{array}$ & $\begin{array}{l}34,5 \\
65,5\end{array}$ & $1694 \pm 177$ \\
\hline 2 & 8 & $56,6 \pm 7,4$ & $\begin{array}{l}5 \mathrm{M} \\
3 \mathrm{~F}\end{array}$ & $\begin{array}{l}62,5 \\
37,5\end{array}$ & $8,1 \pm 1,60 \%$ & $8,0 \pm 1,43 \%$ & $8 \mathrm{RDP}$ & 100 & $1658 \pm 158$ \\
\hline
\end{tabular}

$\mathrm{n}=$ amostra; $\mathrm{M}=$ sexo masculino; $\mathrm{F}=$ sexo feminino; $\mathrm{Hb}_{\mathrm{A1c}}=$ hemoglobina glicosilada pré e pós tratamento; $\mathrm{RD}=$ retinopatia diabética, classificação baseada no $\mathrm{ETDRS}$ $\mathrm{RDP}=$ retinopatia diabética proliferativa; $\mathrm{RDNP}=$ retinopatia diabética não proliferativa muito grave

\begin{tabular}{|c|c|c|c|c|c|c|c|}
\hline Grupo & $\mathbf{n}$ & OCT* (Pré) & $\mathrm{OCT}^{*}\left(1^{\mathrm{a}} \mathrm{sem}\right)$ & OCT* (1ํ mês) & OCT* (3ํ mês) & AV (pré) & AV (3ํำs) \\
\hline 1 & 29 & $198,4 \pm 28,05$ & $223,6 \pm 39,47$ & $224,3 \pm 35,83$ & $222,4 \pm 32,85$ & $0,95 \pm 0,06$ & $0,94 \pm 0,07$ \\
\hline 2 & 8 & $318,9 \pm 75,55$ & $375,1 \pm 65,42$ & $356,2 \pm 87,56$ & $356,7 \pm 50,41$ & $0,56 \pm 0,13$ & $0,36 \pm 0,11$ \\
\hline
\end{tabular}

perda visual 3 meses após o tratamento comparado com o grupo sem edema. Apesar do tamanho da amostra ser diferente, os grupos não apresentaram diferenças significantes na idade, sexo e no controle do diabetes mellitus $\left(\mathrm{Hb}_{\mathrm{Alc}}\right)$; o grupo 2 teve apenas 8 pacientes, no entanto, houve uma tendência similar no comportamento das variáveis analisadas durante todo seguimento, não havendo dispersão importante nos resultados. Um fator limitante para avaliar a AV foi a utilização da tabela de Snellen, idealmente deveria ser empregada a do
ETDRS para detectar pequenas variações com menor risco de erros.

As alterações inflamatórias e do fluxo sanguíneo retiniano podem ser responsáveis pelo mecanismo patogênico de edema macular após a laserterapia ${ }^{(7)}$. A fisiopatologia desta alteração ainda permanece incerta, mas estudos mostram que a destruição térmica na panfotocoagulação induz inflamação no tecido retiniano e algumas citocinas relacionadas ao processo inflamatório (interleucinas 6 e 8) e o óxido nítrico estão aumen- 


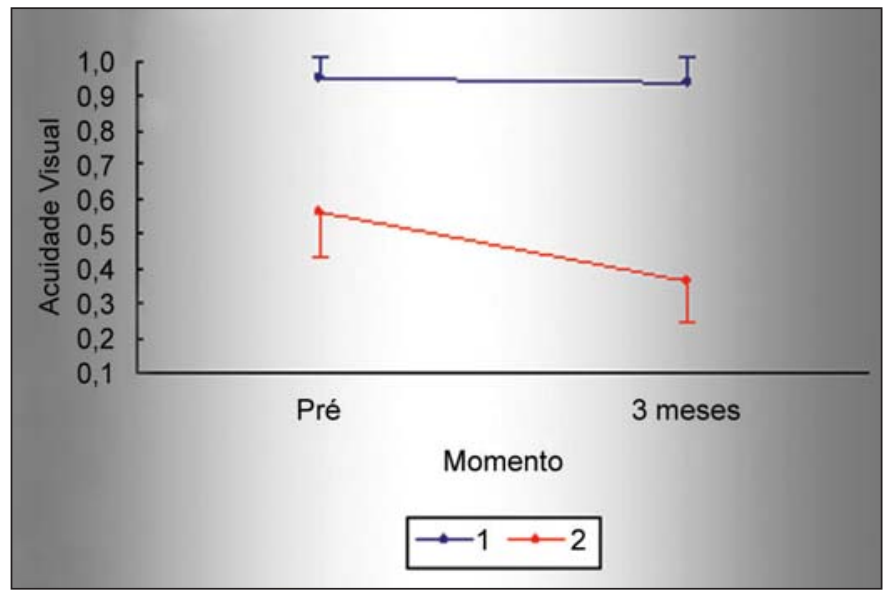

Gráfico 1 - Comparativo da acuidade visual, pré e pós panfotocoagulação, de portadores de retinopatia diabética sem edema macular (Grupo 1) e com edema (Grupo 2). Hospital das Clínicas da Faculdade de Medicina da Universidade de São Paulo - 2005.

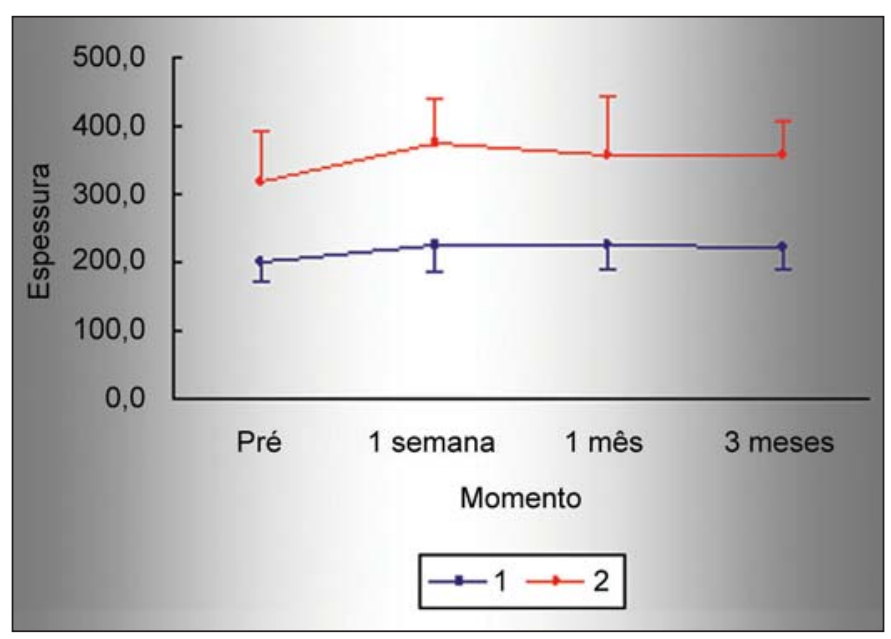

Gráfico 2 - Comparativo de espessura macular, pré e pós panfotocoagulação, de portadores de retinopatia diabética sem edema macular (Grupo 1) e com edema (Grupo 2). Hospital das Clínicas da Faculdade de Medicina da Universidade de São Paulo - 2005.

tados após panfotocoagulação, podendo levar ao edema macular após este tratamento ${ }^{(21-22)}$. A quebra da barreira hematoaquosa foi evidenciada utilizando fotômetro a laser após panfotocoagulação retiniana, sendo mais pronunciada nos primeiros 10 dias do tratamento, apesar de ser demonstrada até 3 meses, de maneira mais branda ${ }^{(23)}$. O tempo de seguimento de 3 meses, determinado para o presente estudo, foi devido à evidência da quebra da barreira no estudo supracitado.

Com estas hipóteses fisiopatológicas e os resultados deste estudo, sugere-se pesquisas de coadjuvantes no manejo de portadores de RD e EMCS que serão submetidos à panfotocoagulação, evitando piora do edema macular e perda visual após tratamento. No entanto, outros trabalhos com maiores casuísticas e tempo de follow-up devem substanciar as evidências das alterações morfológicas e funcionais, en- contradas nesses portadores de RD e EMCS após panfotocoagulação.

\section{CONCLUSÃO}

Evidenciou-se que edema macular na $\mathrm{RD}$, prévio à panfotocoagulação, influencia na espessura macular e na AV póstratamento. Observou-se variação acentuada na espessura macular e perda visual nos portadores de edema macular. Naqueles submetidos à panfotocoagulação e que não tinham edema, observou-se aumento na espessura macular ao longo do seguimento, porém sem repercussões funcionais.

\section{ABSTRACT}

Purpose: To compare the visual acuity and macular thickness in patients with diabetic retinopathy presenting and not presenting macular edema before treatment and submitted to argon laser retinal panphotocoagulation. Methods: Patients were included and classified into two groups before the treatment. All patients were submitted to ophthalmological examination and optical coherence tomography, following panphotocoagulation. The results were evaluated by measuring visual acuity at the end and by optical coherence tomography after one week, one and three months following the treatment. Results: The sample was composed of 37 patients (37 eyes). Group 1 included 29 patients with diabetic retinopathy without macular edema; Group 2 included 8 patients with macular edema. The two groups were similar regarding gender, age, and the mean of argon laser spots treatment. The groups showed differences in visual acuity $(\mathrm{p}<0.001)$ and Group 2 patients presented decrease of visual acuity three months after the treatment $(\mathrm{p}<0.001)$. Group 2 patients showed a greater mean of macular thickness measurements on optical coherence tomography evaluation. Conclusion: Variation of the macular thickness as well as decrease in visual acuity was present in the patients with macular edema previously to panphotocoagulation. Only minimal increase in macular thickness was observed in the absence of macular edema.

Keywords: Diabetic retinopathy; Diabetic retinopathy/therapy; Macular edema, cystoid; Laser coagulation; Diabetes mellitus/complications; Visual acuity; Tomography, optical coherence

\section{REFERÊNCIAS}

1. Fundus photographic risk factors for progression of diabetic retinopathy. ETDRS report $\mathrm{n}^{\circ}$ 12. Early Treatment Diabetic Retinopathy Study Research Group Ophthalmology. 1991;98(5 Suppl):823-33.

2. Treatment techniques and clinical guidelines for photocoagulation of diabetic macular edema. Early Treatment Diabetic Retinopathy Study Report Number 2. Early Treatment Diabetic Retinopathy Study Research Group. Ophthalmology. 1987;94(7):761-74. 
3. Photocoagulation for diabetic macular edema: Early Treatment Diabetic Retinopathy Study Report $\mathrm{n}^{\circ}$ 4. The Early Treatment Diabetic Retinopathyh Study Research Group. Int Ophthalmol Clin. 1987;27(4):265-72.

4. Indications for photocoagulation treatment of diabetic retinopathy: Diabetic Retinopathy Study Report n. 14. The Diabetic Retinopathy Study Research Group. Int Opthalmol Clin. 1987;27(4):239-53.

5. Four risk factors for severe visual loss in diabetic retinopathy. The third report from the Diabetic Retinopathy Study. The Diabetic Retinopathy Study Research Group. Arch Ophthalmol. 1979;97(4):654-5.

6. Diabetic Retinopathy Study. Report Number 6. Design, methods, and baseline results. Report Number 7. A modification of the Airline House classification of diabetic retinopathy. Prepared by the Diabetic Retinopathy. Invest Ophthalmol Vis Sci. 1981;21(1 Pt 2):1-226.

7. McDonald HR, Schartz H. Macular edema following panretinal photocoagulation. Retina. 1985;5(1):5-10

8. McDonald HR, Schartz H. Visual loss following panretinal photocoagulation for proliferative diabetic retinopathy. Ophthalmolgy. 1985;92(3):388-93.

9. Myers SM. Macular edema after scatter laser photocoagulation for proliferative diabetic retinopathy. Am J Ophthalmol. 1980;90(2):210-6.

10. Kleiner RC, Elman MJ, Murphy RP, Ferris FL $3^{\text {rd }}$. Transient severe visual loss after panretinal photocoagulation. Am J Ophthalmol. 1988;106(3):298-306.

11. Ferris FL $3^{\text {rd }}$, Podgor MJ, Davis MD. Macular edema in Diabetic Retinopathy Study patients. Diabetic Retinopathy Study Report Number 12. Ophthalmology. 1987;94(7):754-60.

12. Editorial: The diabetic retinopathy study. Arch Ophthalmol. 1973;90(5):347-8.

13. Rosner B. Fundamentals of Biostatistics. $2^{\text {nd }}$ ed. Boston, PWS; 1986, p. 584.

14. Timm N.H. Multivariate analysis with applications in educations and psychology. Monterrey, CA: Brooks/Cole; 1975. p.687.

15. Tsujikawa A, Kiryu J, Dong J, Yasukawa T, Suzuma I, Takagi H, et al.
Quantitative analysis of diabetic macular edema after scatter laser photocoagulation with the scanning retinal thickness analyzer. Retina. 1999;19(1):59-64.

16. Shimura M, Yasuda K, Nakazawa T, Kano T, Ohta S, Tamai M. Quantifying alterations of macular thickness before and after panretinal photocoagulation in patients with severe diabetic retinopathy and good vision. Ophthalmology. 2003;110(12):2386-94.

17. Shimura M, Yasuda K, Nakazawa T, Tamai M. Visual dysfunction after panretinal photocoagulation in patients with severe diabetic retinopathy and good vision. Am J Ophthalmol. 2005;140(1):8-15. Comment in: Am J Ophthalmol. 2005;140(1):127-7; Am J Ophthalmol. 2006;14(2):422; author reply 422-3.

18. Otani T, Kishi S, Maruyama Y. Patterns of diabetic macular edema with optical coherence tomography. Am J Ophthalmol. 1999;127(6):688-93.

19. Yang CS, Cheng CY, Lee FL, Hsu WM, Liu JH. Quantitative assessment of retinal thickness in diabetic patients with and without clinically significant macular edema using optical coherence tomography. Acta Ophthalmol Scand. 2001;79(3):266-70.

20. Browning DJ, McOwen MD, Bowen RM Jr, O'Marah TL. Comparison of the clinical diagnosis of diabetic macular edema with diagnosis by optical coherence tomography. Ophthalmology. 2004;111(4):712-5. Comment in: Ophthalmology. 2005;112(4):734-5; author reply 735 .

21. Nonaka A, Kiryu J, Tsujikawa A, Yamashiro K, Nishijima K, Kamizuru H, et al. Inflammatory response after scatter laser photocoagulation in nonphotocoagulated retina. Invest Ophthalmol Vis Sci. 2002;43(4):1204-9.

22. Er H, Doganay S, Turkoz Y, Cekmen M, Daglioglu MC, Gunduz A, et al. The levels of cytokines and nitric oxide in rabbit vitreous humor after retinal laser photocoagulation. Ophthalmic Surg Lasers. 2000;31(6):479-83.

23. Larsson LI, Nuija E. Increased permeability of the blood-aqueous barrier after panretinal photocoagulation for proliferative diabetic retinopathy. Acta Ophthalmol Scand. 2001;79(4):414-6. 